\title{
The Optical Anisotropy of Wormlike Chains
}

\author{
DIETMAR PORSCHKE and GERHARD NOLTE
}

Max-Planck Institut für biophysikalische Chemie, 34 Göttingen, Federal Republic of Germany

The optical anisotropy of macromolecules like DNA usually is analyzed without explicit consideration of their flexibility. ${ }^{1-6}$ In some cases this simplified approach has been justified by the argument that the chain length of the molecules under investigation did not exceed the persistence length. A recent model calculation ${ }^{7}$ has demonstrated, however, that the effect of chain flexibility on the optical anisotropy (electric dichroism or birefringence) is considerable even for chains shorter than the persistence length. The model was developed for molecules that are inherently straight but subject to thermal bending in a simple continuous bending mode, resulting in bent forms corresponding to circular arcs. This model is still treatable with a moderate degree of computation and has been incorporated into an orientation function including the effect of field-induced stretching. Since the model does not consider the full degree of bending freedoms, it has been proposed as an approximation for chains below the persistence length. For a final assessment of its validity the optical anisotropy should be calculated for wormlike chains without any restriction on the bending mode. The optical anisotropy has been given in the literature for the case of long wormlike chains ${ }^{8,9}$; however, these results cannot be used for the analysis of experimental data obtained for chains in the range of the persistence length, which is of particular interest for investigations of DNA restriction fragments. For this purpose we have used Monte Carlo simulations. The results of the calculations show that the weakly bent rod model underestimates the effect of bending on the optical anisotropy, but nevertheless provides a very reasonable approximation-even for chains longer than the persistence length.

\section{SIMULATION PROCEDURE}

Wormlike chains were simulated as reported by Hagerman and $\mathrm{Zimm}^{10}$ and thus we present the essentials of this simulation only very briefly. The sequence of segment vectors is described by sequences of angles $\phi_{i}$ and $\theta_{i}$. The $\phi$ is chosen with equal probability between 0 and $2 \pi$ (using

(C) 1990 John Wiley \& Sons, Inc.

CCC 0006-3525/90/13-141289-02 \$04.00

Biopolymers, Vol. 30, 1289-1290 (1990) the IMSL routine DRNUN), whereas the probability $f(\theta)$ for a given value of $\theta$ is represented by

$$
f(\theta)=C \cdot e^{-\left(p \cdot \theta^{2} / 2 d\right)} \cdot \sin \theta
$$

with $p$ the persistence length, $d$ the distance between adjacent elements, and $C$ the scaling factor (calculation as by Hagerman and $\mathrm{Zimm}^{10}$; normal distribution for individual orthogonal angular displacements by IMSL routine DRNNOA). The sequence of centers of beads $P(i)$ corresponds to a sequence of coordinate systems $K(i): K(i$ $+1)$ is generated from $K(i)$ by counterclockwise rotation around the $z$ axis by the angle $\phi_{i}$ and subsequent clockwise rotation around the new $y$ axis by angle $\theta_{i}$. As center of the $i+1$ st bead we take the point $P(i+1)=(0,0, \mathrm{~d})$ $+P(i)$-in the coordinates of $K(i+1)$. The results obtained with our coordinate system are equivalent to those obtained with other coordinate systems. The matrix $M(i)$ for transformation from $K(i+1)$ to $K(i)$ is given by

$$
\begin{array}{lll}
\cos \left(\phi_{i}\right) \cdot \cos \left(\theta_{i}\right) & -\sin \left(\phi_{i}\right) & \cos \left(\phi_{i}\right) \cdot \sin \left(\theta_{i}\right) \\
\sin \left(\phi_{i}\right) \cdot \cos \left(\theta_{i}\right) & \cos \left(\phi_{i}\right) & \sin \left(\phi_{i}\right) \cdot \sin \left(\theta_{i}\right) \\
-\sin \left(\theta_{i}\right) & 0 & \cos \left(\theta_{i}\right)
\end{array}
$$

Applying $M(i), M(i-1), \ldots, M(0)$-in this order we obtain the coordinates of $P(i+1)$ in the initial system $K(0)$. The chain is represented by the centers of their beads $P(0), \ldots, P(\mathrm{n}) . P(0)$ is fixed to $(0,0,0)$ and $P(1)$ to $(0,0, d)$ without loss of general validity. We have checked our ensembles of wormlike chains by calculation of the reduced mean-squared end-to-end distances $\left\langle h^{2}\right\rangle /$ $l^{2}$ and found agreement with the standard expression ${ }^{11}$

$$
\left\langle h^{2}\right\rangle / l^{2}=(2 p / l)[1-p / l+(p / l) \exp (-l / p)]
$$

For an evaluation of the optical anisotropy we have to define the direction of preferential alignment. When the molecules are aligned by an electric field, the maximum value of the induced dipole moment will be in the direction of maximal extension, which corresponds to the end-toend vector-at least for relatively short chains. A corresponding argument holds for orientation of molecules induced by flow. Thus, for the evaluation of the linear dichroism, we have calculated the contributions of individual 


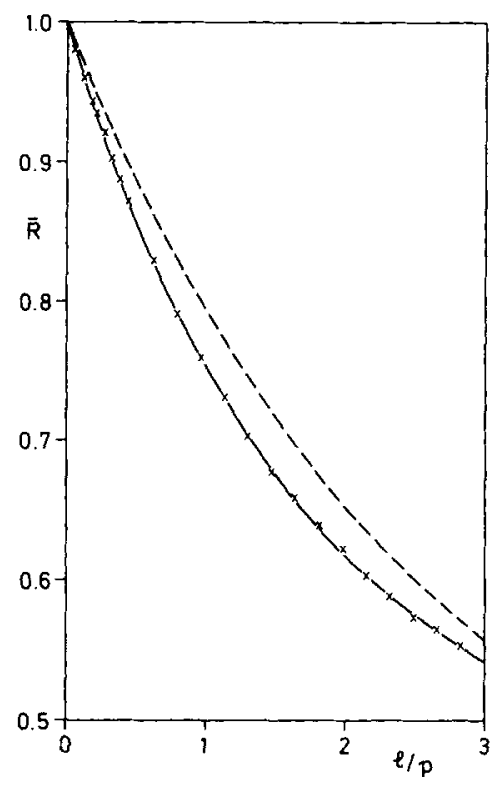

Figure 1. Relative optical anisotropy $\bar{R}$ as a function of the ratio $l / p$ ( $l$ is the chain length and $p$ the persistence length). The lower line represents the polynomial fit to the points $(x)$ simulated according to the wormlike chain; the upper line (dashed) represents the weakly bent rod model.

transition dipole moments in the direction of the end to end vector according to

$$
R(i)=\frac{\langle P(i)-P(i-1), E\rangle^{2}}{\langle P(i)-P(i-1), P(i)-P(i-1)\rangle}
$$

$E$ represents the unit vector in end-to-end direction and the angle brackets the usual scalar product.

The sum $R$ of $R(i)$ gives the dichroism (or the birefringence) of a given wormlike chain relative to that of the same chain in its straight form. We have calculated average ratios $\bar{R}$ for ensembles of 10,000 independent chains of given contour length $l$ at fixed persistence length $p$. For ensembles of this size the standard deviation of the average ratios $\bar{R}$ was approximately \pm 0.001 . This accuracy was verified by calculation of independent ensembles. As should be expected, $\bar{R}$ decreases with increasing $l / p$ values (cf. Figure 1). We have fitted our numerical results by the polynomial

$$
\begin{aligned}
\bar{R}=1-0.3222(l / p)+0.08504(l / p)^{2} & \\
& -0.009521(l / p)^{3}
\end{aligned}
$$

A comparison with the corresponding results obtained by the weakly bent rod model shows that the optical anisotropy of the wormlike chain is somewhat lower. A maximal deviation is observed at an $l / p$ ratio of about 1.5 , where the optical anisotropy of the weakly bent rod is $6 \%$ higher than that for the wormlike chain. In summary, the agreement is quite satisfactory and thus the weakly bent rod model can be used as a very reasonable approximation for the description of the optical anisotropy of wormlike chains-even for ratios of the chain length to the persistence length up to 3. Thus, in the case of DNA double helices the simple model may be used at least for fragments up to a few hundreds of base pairs.

We thank Drs. Jan Antosiewicz and John McCaskill for reading the manuscript. For our computations we used the facilities of the Gesellschaft für wissenschaftliche Datenverarbeitung $\mathrm{mbH}$, Göttingen.

\section{REFERENCES}

1. Ding, D., Rill, R. \& Van Holde, K. E. (1972) Biopolymers 11, 2109.

2. Emonds-Alt, X., Houssier, C. \& Fredericq, E. (1979) Biophys. Chem. 10, 27.

3. Sokerov, S. \& Weil, G. (1979) Biophys. Chem. 10, 161.

4. Hogan, M., Dattagupta, N. \& Crothers, D. M. (1978) Proc. Natl. Acad. Sci. USA 75, 195.

5. Lee, C. H. \& Charney, E. (1982) J. Mol. Biol. 161, 289.

6. Diekmann, S., Hillen, W., Jung, M., Wells, R. D. \& Porschke, D. (1982) Biophys. Chem. 15, 157.

7. Porschke, D. (1989) Biopolymers 28, 1383.

8. Kuhn, W. \& Grün, F. (1942) Kolloid Zeitschr. 101, 248.

9. Wilson, R. W. \& Schellman, J. A. (1977) Biopolymers 16, 2143 .

10. Hagerman, P. J. \& Zimm, B. H. (1981) Biopolymers $20,1481$.

11. Bloomfield, V. A., Crothers, D. M. \& Tinoco, I. (1974) Physical Chemistry of Nucleic Acids, Harper \& Row, New York, p. 160.

Received February 23, 1990

Accepted July 5, 1990 